Check for updates

Cite this: RSC Adv., 2017, 7, 32518

Received 14th April 2017

Accepted 20th June 2017

DOI: $10.1039 / c 7 r a 04228 k$

rsc.li/rsc-advances

\title{
Molecular interactions between DOPA and surfaces
with different functional groups: a chemical force \\ Molecular interactions between DOPA and surfaces
with different functional groups: a chemical force microscopy study $\dagger$
}

\author{
Wei Zhang, ${ }^{\text {ab }}$ Hui Yang, ${ }^{\text {*a }}$ Fanghui Liu, ${ }^{a}$ Ting Chen, ${ }^{a}$ Guangxin $\mathrm{Hu}^{\mathrm{ab}}$ Donghong Guo, ${ }^{\mathrm{c}}$ \\ Qingfeng $\mathrm{Hou}^{\mathrm{c}}{ }^{\mathrm{X}} \mathrm{Xu} \mathrm{Wu},{ }^{d} \mathrm{Yu} \mathrm{Su}^{\star b}$ and Jinben Wang (D) *a
}

\begin{abstract}
The adhesion of mussel foot proteins (Mfps) to a variety of surfaces has been widely investigated, but the mechanisms behind the mussel adhesion to surfaces with different properties are far from being understood. Most of Mfps contain a significant amount of 3,4-dihydroxyphenylalanine (DOPA) which is considered to be responsible for the strong wet adhesion. In the present work, self-assembled monolayers (SAMs) were prepared as a series of model surfaces with variable functional groups. DOPA-surface interactions were investigated using chemical force microscopy (CFM) for the first time, in which an atomic force microscope (AFM) tip was chemically modified with DOPA terminated groups. The ability of DOPA to adhere to different surfaces with variable wettability was compared, showing that DOPA behaves with the strongest and weakest adhesion to $\mathrm{C}_{6} \mathrm{H}_{5}$ - and $\mathrm{OH}$-terminated surfaces, respectively. The interaction strength of DOPA at different surfaces does not always increase with the increase of surface wettability, because the hydrophobic interaction does not play a decisive role in DOPA adhering to surfaces. By the use of classical and extended Derjaguin-Landau-Verwey-Overbeek (DLVO) theories, the contribution of non-DLVO forces was isolated. We found out DOPA can adhere to each surface functional group, since DOPA residues containing o-hydroxy or aromatic rings alone can control the adhesion process, and the aromatic ring is oriented perpendicularly or parallel to the surface. This study served as a basis for understanding the relationship between DOPA adhesion mechanisms and different wet surfaces, representing important concepts for the design of bioadhesive materials and anti-adhesion surfaces.
\end{abstract}

\section{Introduction}

Marine mussels are a well-known biological family due to their remarkable ability to stick to wet surfaces and achieve longlasting adhesion. ${ }^{1-7}$ On the one hand, the undesired colonization has serious impacts, in particular in promoting ship surface corrosion, increasing fuel consumption, and hampering the accuracy and reliability of underwater sensors. ${ }^{8,9}$ On the other hand, the desired adhesion can be developed as excellent biomimetic adhesives of soft tissue, which promotes tissue regeneration and minimizes surgery time by replacing common surgical procedures. ${ }^{\mathbf{1 0 1 1}}$ The reason why mussels are good at

${ }^{a}$ Key Laboratory of Colloid, Interface and Chemical Thermodynamics, Institute of Chemistry, Chinese Academy of Sciences, Beijing 100190, P. R. China. E-mail: yanghui@iccas.ac.cn; jbwang@iccas.ac.cn

${ }^{b}$ School of Chemistry and Materials Science, Heilongjiang University, Harbin 150080, P. R. China. E-mail: suyu-0451@163.com

'Key Laboratory of Oilfield Chemistry, Research Institute of Petroleum Exploration and Development (RIPED), CNPC, Beijing 100083, P. R. China

${ }^{d}$ College of Chemistry and Chemical Engineering, Guangzhou University, Guangzhou 510006, P. R. China

$\dagger$ Electronic supplementary information (ESI) available. See DOI: $10.1039 / \mathrm{c} 7 \mathrm{ra} 04228 \mathrm{k}$ attaching to virtually any material underwater, whether natural or synthetic, is that mussels produce a bundle of threads tipped with adhesive pads, known collectively as byssus, composing of different types of mussel foot proteins (Mfps). ${ }^{12,13}$ 3,4-Dihydroxyphenylalanine (DOPA) is a unique amino acid widely found in Mfps: mfp-3 and mfp-5 have been found to have a high content, 20-30 mol\%, of DOPA that plays a key role in mussel adhesion., 14-16 However, the contribution of DOPA in the adhesion process of mussels at surfaces has still been a challenging problem. ${ }^{17-21}$ Till now, there has been only a little knowledge about the interactions between DOPA and inorganic surfaces or organic surfaces (including polytetrafluoroethylene, polystyrene, and high-density polyethylene) underwater. $^{\mathbf{1 4 , 2 2}}$ While in the natural world, pristine surfaces are always covered by various organic films in a dynamic and patchy process, ${ }^{23,24}$ and therefore, it is necessary to understand DOPA binding mechanisms to such fouled films due to the great importance in marine biofouling and the biocompatibility of biomaterials. Besides, there is currently a lack of knowledge about how material factors, such as surface chemistry and wettability, can control protein adhesion/morphology. ${ }^{25}$

With this in mind, we focused on how changing intricate surface chemistry can influence DOPA adhesion, and fabricated 
a series of model surfaces (self-assembled monolayers (SAMs) which provide an easy and controlled way to create surfaces with different functional groups), and investigated the interactions of DOPA-surface as a function of surface chemistry. Although surface force apparatus (SFA) has been used to measure the macroscopic dissociation of two surfaces adhered by mussel proteins, yet the method did not reveal the adhesive interaction of DOPA-SAMs surface. ${ }^{\mathbf{1 9 , 2 6 - 3 2}}$ In order to gain further insight into the molecular interactions between DOPA molecules and the series of SAM surfaces, chemical force microscopy (CFM) was introduced to the present study, in which the contribution of DOPA in mussel adhesion was successfully isolated. With this approach, we were able to quantitatively measure the interactions between DOPA and seven different wettability surfaces systematically, and find the relationship between DOPA adhesion and surface wettability, as well as reveal which surfaces have the potential to be applied to the anti-adherent material and which have the potential to be used in making adhesive materials. By the use of classical and extended Derjaguin-Landau-Verwey-Overbeek (DLVO) theories, the contribution of non-DLVO forces was evaluated. DOPA adhered to various functional groups through different interactions, from which the most important binding types were concluded. This study provides novel strategies for the design of antifouling and adhesive surfaces underwater.

\section{Experimental section}

\section{Preparation of self-assembled monolayers (SAMs)}

Silicon wafers were cut into square strips and soaked in piranha solution $\left(\mathrm{H}_{2} \mathrm{SO}_{4} / \mathrm{H}_{2} \mathrm{O}_{2}=3: 1 \mathrm{v}: \mathrm{v}\right)$ for $30 \mathrm{~min}$ at $85^{\circ} \mathrm{C}$, and then thoroughly rinsed with Millipore Milli-Q grade water (18.2 $\mathrm{M} \Omega$ $\mathrm{cm})$. Gold substrates were prepared by first evaporating $5 \mathrm{~nm}$ of $\mathrm{Cr}$ adhesion layer on cleaned silicon wafers, and then $50 \mathrm{~nm}$ of $\mathrm{Au}$ under a high vacuum through an electron beam evaporator (Peva-600E, Campro, Taiwan). Prior to SAM formation on these substrates, they were cleaned by dipping them for $1 \mathrm{~min}$ in piranha solution, and then rinsed copiously with Millipore Milli-Q grade water. SAMs-functionalized surfaces were prepared as per the established protocols described previously. ${ }^{33-35}$ The freshly clean substrates were immersed in ethanolic solutions of 11-mercapto-1-undecanol $\left(\mathrm{SH}-\left(\mathrm{CH}_{2}\right)_{11} \mathrm{OH}\right.$, Aldrich), 11-mercaptoundecanoic acid $\left(\mathrm{SH}-\left(\mathrm{CH}_{2}\right)_{10} \mathrm{COOH}, \mathrm{J} \& \mathrm{~K}\right.$ Scientific Ltd), 11-amino-1-undecanethiol, hydrochloride (SH$\left(\mathrm{CH}_{2}\right)_{11} \mathrm{NH}_{2} \mathrm{HCl}$, Aldrich), $1 \mathrm{H}, 1 \mathrm{H}, 2 \mathrm{H}, 2 \mathrm{H}$-perfluorodecanethiol (SH- $\left(\mathrm{CH}_{2}\right)_{2}\left(\mathrm{CF}_{2}\right)_{7} \mathrm{CF}_{3}$, J\&K Scientific Ltd), octadecanethiol (SH$\left(\mathrm{CH}_{2}\right)_{17} \mathrm{CH}_{3}$, Alfa Aesar), and 10-phenyldecylmercaptan (SH$\left(\mathrm{CH}_{2}\right)_{10} \mathrm{C}_{6} \mathrm{H}_{5}$, Aldrich) at the concentration of $1 \mathrm{mM}$ for $24 \mathrm{~h}$, respectively. The substrates were thoroughly rinsed by ethanol to remove physisorbed thiol from them and dried with pure nitrogen stream.

\section{Surface characterization of SAMs}

X-ray photoelectron spectroscopy (XPS) was performed on Thermo Scientific ESCALab 250Xi using $200 \mathrm{~W}$ monochromated $\mathrm{Al} \mathrm{K} \alpha$ radiation. The $500 \mu \mathrm{m}$ X-ray spot was used for XPS analysis. The base pressure in the analysis chamber was about 3 $\times 10^{-10}$ mbar. Typically the hydrocarbon C 1 s line at $284.8 \mathrm{eV}$ from adventitious carbon is used for energy referencing.

The contact angle (CA) was measured through a Kruss DSA CA goniometer (Germany) equipped with a dispensing needle. The sessile droplet with a volume of $5 \mu \mathrm{L}$ was formed by fixing the needle and approaching the substrate in parallel with the needle direction at a slow feed rate. All the measurements were performed in air at ambient temperature.

Surface potentials of the series of surfaces (size in $20 \mathrm{~mm}$ long, $10 \mathrm{~mm}$ wide, and $1 \mathrm{~mm}$ thick) were measured on a surpass electrokinetic analyzer (Anton Parr, Austria). Determination of the potentials was based on the measurement of streaming potential and current. In the streaming potential measurements, a water buffered with $25 \mathrm{mM}$ Tris $\mathrm{HCl}$ solution at pH 5.5 was pumped to flow along the surface of the sample and the potential was measured based on the following relationship: ${ }^{36}$

$$
\zeta=\frac{\mathrm{d} U}{\mathrm{~d} P} \times \frac{\eta}{\varepsilon \times \varepsilon_{0}} \times K
$$

where $\zeta$ is zeta potential; $\mathrm{d} U / \mathrm{d} P$ denotes the slope of streaming potential versus pressure; $\eta, \varepsilon_{0}, \varepsilon$, and $K$ represent electrolyte viscosity, vacuum permittivity, dielectric constant of the buffer, and its conductivity, respectively. The streaming potential or alternatively the streaming current was detected by the electrodes placed at both sides of the sample, as shown in Table S1. $\dagger$

Surface topography of SAM-functionalized surfaces was measured via AFM in a peakforce tapping mode (Dimension Fast scan, Bruker Corp.). AFM measurements were conducted using a probe made of silicon nitride (Fast Scan-B, Bruker Corp.). The topography images were performed on a scan area of $1 \mu \mathrm{m} \times 1 \mu \mathrm{m}$ at a speed of $3.91 \mathrm{~Hz}$.

\section{AFM tip modification and characterization}

AFM silicon nitride tips (HLCT, Bruker Corp.) were cleaned in an $\mathrm{O}_{2}$ plasma (Evactron 25, XEI Scientific, Inc, USA) for $15 \mathrm{~min}$ and then subsequently transferred to a piranha solution for 30 min (reaction I in Fig. 1), in order to remove impurities and generate a hydroxyl layer on the tip surface. After gently rinsing with Mili-Q water, the tip was transferred into $0.5 \mathrm{mM}$ silanePEG-NH ${ }_{2}\left(M_{\mathrm{W}} 3400\right.$, Nanocs $)$ in toluene solution for $6 \mathrm{~h}$, resulting in an aminosilane-functionalized tip (reaction II). The tip was thoroughly rinsed with large amounts of toluene solution to remove unreacted molecules. Then the cantilever was stored in an oven at $110{ }^{\circ} \mathrm{C}$ for $10 \mathrm{~min}$ to stabilize the silane conjugation. Next, the tip was immersed in a $N, N$-dimethylformamide (DMF) solution containing $\mathrm{N}$-methylmorpholine, 2-(7-aza-1 $H$-benzotriazole-1-yl)-1,1,3,3-tetramethyluronium hexafluorophosphate (HATU) and $N$-Boc-DOPA for $3 \mathrm{~h}$, followed by coupling of $N$-Boc-DOPA to the liberated amine in solution, as shown in reaction III. After the modification, $N$-Boc-DOPA was end-tethered to PEG, and Boc protected amine groups remaining in place to avoid electrostatic interactions. ${ }^{\mathbf{1 4}}$

In order to confirm that the chemical modification of the AFM tip was successful, we functionalized a silicon substrate with Boc-DOPA using exactly the same procedure that we used 

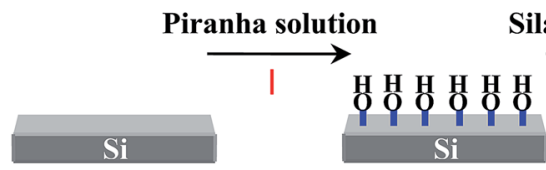

s

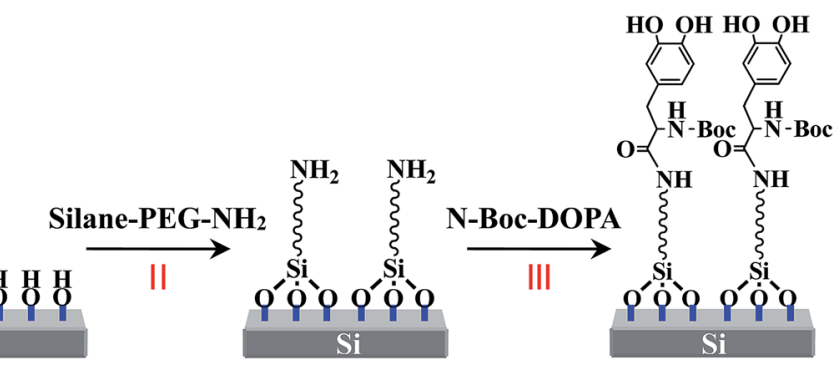

Fig. 1 Schematic of AFM tip preparation.

for the tip. We characterized the resulting surface at each step of the procedure through XPS and CA methods (Fig. S1 $\dagger$ ). C 1s peaks at around $284.8 \mathrm{eV}$ and $286.5 \mathrm{eV}$ were observed, which were assigned to $\mathrm{C}-\mathrm{C}$ and $\mathrm{C}-\mathrm{O}$, respectively. The peak of $\mathrm{N} 1 \mathrm{~s}$ at $\sim 400 \mathrm{eV}$ was observed. The change of the test element ( $\mathrm{C}$ and $\mathrm{N}$ ) content was consistent with the change of the surface chemical composition along with the modification process. The contact angle also increased with the modification from $17^{\circ}$ to $28^{\circ}$, further illustrating the success of the modification process. In the absence of DOPA, the PEG itself interacted weakly with surfaces as compared with the DOPA-functionalized AFM tips, as shown in Fig. S2. $\dagger$ In the test of adhesion interactions between DOPA and each substrate, a small amount of free amino groups had little effect on the interaction results, which can be neglected even if there were some unsuccessful grafting of DOPA.

\section{Force-measuring technique}

The fluid chamber was filled in with $1.5 \mathrm{~mL}$ of buffer containing $25 \mathrm{mM}$ Tris $\mathrm{HCl}$ at $\mathrm{pH}$ 5.5. Atomic force microscopic (AFM, HLCT, Bruker Corp.) experiments were conducted after allowing the system to equilibrate for $30 \mathrm{~min}$. AFM silicon nitride cantilevers with silicon nitride tips (MLCT, Bruker Corp.) were used in all experiments. The spring constants of the tips were in the range of $0.05-0.07 \mathrm{~N} \mathrm{~m}^{-1}$ calibrated by thermal fluctuation method. In order to ensure that the DOPA used for testing was not oxidized, the DOPA functionalized AFM tip was immersed in $1 \mathrm{mM}$ of ascorbic acid solution for $30 \mathrm{~min}$ before each AFM experiment. ${ }^{22,37}$ In a typical experiment, a cantilever was brought to the surface at a constant speed of $1000 \mathrm{~nm} \mathrm{~s}^{-1}$ and held on the surface at constant forces of 1-2 $\mathrm{nN}$ to allow a sufficient contact of DOPA-modified tip and functional surface. Then the cantilever was moved back at the same speed to break DOPA-surface interactions. We used the same DOPAterminated tip and made all the AFM measurements in the same conditions (such as spring constant, constant speed, et al.) to minimize the influence of DOPA modification density on DOPA binding strength. All AFM force measurements were carried out at room temperature.

\section{Theory and calculation}

\section{DLVO model}

Total DLVO interaction force $\left(F_{\text {total }}\right)$ between two interacting surfaces was calculated as the sum of Lifshitz-van der Waals
$\left(F_{\text {van der Waals }}\right)$ and electrostatic interactions ( $\left.F_{\text {electrostatic }}\right)$, as shown in eqn (2): ${ }^{38}$

$$
F_{\text {total }}=F_{\text {van der Waals }}+F_{\text {electrostatic }}
$$

The expression for the van der Waals force between AFM tip and substrate, based on Hamaker's approach and Derjaguin's approximation used in this study, is given by eqn (3): $:^{39}$

$$
F_{\text {van der Waals }}(h)=\frac{A}{6}\left[-\frac{a}{h^{2}}-\frac{a}{(h+2 a)^{2}}+\frac{2 a}{h(h+2 a)}\right]
$$

where $a$ is the radius of AFM tip ( $\sim 20 \mathrm{~nm}) ; h$ is the surface-tosurface distance between substrate and AFM tip; $A$ is the effective Hamaker constant, which is the key point in calculating van der Waals forces, as expressed by eqn (4): ${ }^{40,41}$

$$
\begin{aligned}
A= & \frac{3}{4} k T\left(\frac{\varepsilon_{1}-\varepsilon_{3}}{\varepsilon_{1}+\varepsilon_{3}}\right)\left(\frac{\varepsilon_{2}-\varepsilon_{3}}{\varepsilon_{2}+\varepsilon_{3}}\right)+\frac{3 h}{4 \pi} \int_{v_{1}}^{\infty}\left(\frac{\varepsilon_{1}(\mathrm{iv})-\varepsilon_{3}(\mathrm{iv})}{\varepsilon_{1}(\mathrm{iv})+\varepsilon_{3}(\mathrm{iv})}\right) \\
& \times\left(\frac{\varepsilon_{2}(\mathrm{iv})-\varepsilon_{3}(\mathrm{iv})}{\varepsilon_{2}(\mathrm{iv})+\varepsilon_{3}(\mathrm{iv})}\right) \mathrm{d} v
\end{aligned}
$$

where $\varepsilon_{1}, \varepsilon_{2}$, and $\varepsilon_{3}$ are the static dielectric constants; media 1 and 2 represent AFM tip and substrate, respectively; media 3 stands for water buffered with $25 \mathrm{mM}$ Tris $\mathrm{HCl}$ (pH 5.5); $\varepsilon_{1}$ (iv), $\varepsilon_{2}(\mathrm{iv})$, and $\varepsilon_{3}$ (iv) are the electronic absorption terms; $k$ is the Boltzmann constant, $1.381 \times 10^{-23} \mathrm{~J} \mathrm{~K} \mathrm{~K}^{-1} ; T$ is the Kelvin temperature, $298.15 \mathrm{~K} ; h$ is the plank constant, $6.626 \times 10^{-34}$ J s.

Assuming constant surface potential on the substrates, the well-known Hogg, Healy, and Fuerstenau (HHF) expression for the electrostatic force is used in fitting the data, as shown in eqn $(5)::^{42,43}$

$$
F=\frac{4 \pi R \varepsilon \varepsilon_{0}}{\lambda_{\mathrm{D}}} \frac{\left[\psi_{1} \psi_{2}\left(\mathrm{e}^{k h}+\mathrm{e}^{-k h}\right)-\left(\psi_{1}^{2}+\psi_{2}^{2}\right)\right]}{\left(\mathrm{e}^{k h}-\mathrm{e}^{-k h}\right)^{2}}
$$

where $\varepsilon_{0}\left(8.854 \times 10^{-12} \mathrm{C}^{2} \mathrm{~J}^{-1} \mathrm{~m}^{-1}\right)$ is the permittivity of vacuum, and $\varepsilon(80)$ denotes the relative permittivity of the solution; $\psi_{1}$ and $\psi_{2}$ correspond to the surface potentials of substrate and probe surface, respectively; $h$ is the surface-tosurface distance between substrate and AFM tip; surface potentials are approximated by $\zeta$ potentials $(\mathrm{mV})$ of functionalized surfaces (Table S1 $\dagger$ ); $\lambda_{\mathrm{D}}$ denotes the Debye length as described by eqn (6): $:^{44,45}$

$$
\lambda_{\mathrm{D}}=\sqrt{\frac{\varepsilon \varepsilon_{0} k T}{2 c e^{2}}}
$$


where $e$ is the electron charge $\left(1.602 \times 10^{-19} \mathrm{C}\right)$ and $c$ corresponds to the concentration.

\section{Extended DLVO model}

In the present paper, DLVO theory was extended to provide a more complete interpretation of force-distance experimental data, as expressed in eqn (7): ${ }^{38,46,47}$

$$
F_{\text {total }}=F_{\text {van der Waals }}+F_{\text {electrostatic }}+F_{\text {structrual }}
$$

where $F_{\text {structrual }}$ represents non-DLVO forces, including hydrogen bonding, metal coordination, $\pi$-cation interaction, et al.

$$
F_{\mathrm{s}}=C_{1} \mathrm{e}^{-\left(h-b_{1}\right)^{2} / \lambda_{1}{ }^{2}}+C_{2} \mathrm{e}^{-\left(h-b_{2}\right)^{2} / \lambda_{1}{ }^{2}}+\cdots
$$

where $C$ and $\lambda$ describe coefficient and decay length, respectively; $b$ is a real constant; $h$ denotes separation of tip-substrate. When the fitting profiles were consistent with the experimental profiles, the fitting parameters of EDLVO model were obtained as shown in Table S2.†

\section{Results and discussion}

\section{Surface characterization}

On the series of SAM-terminated surfaces, contact angle of water droplet on such surfaces increases from $\sim 25^{\circ}$ to $\sim 109^{\circ}$, as shown in Fig. 2a-g, implying that the model surfaces vary gradually in water wettability. From Fig. $2 \mathrm{~h}-\mathrm{n}$ (except bare gold surface in Fig. 2k), the high-resolution XPS $2 p$ spectrum of SAMs exhibits a characteristic doublet with red curves peaks at around $162.1 \mathrm{eV}\left(2 \mathrm{p}_{3 / 2}\right)$ and $163.3 \mathrm{eV}\left(2 \mathrm{p}_{1 / 2}\right)$ corresponding to thiolates (RS-) bound to gold. Additionally, a minor doublet component with blue curves peaks at around $163.8 \mathrm{eV}$ and $164.4 \mathrm{eV}$ appears, corresponding to unbound thiol residues but in a relatively low content. ${ }^{48,49} \mathrm{~A}$ peak at around $286.7 \mathrm{eV}$, $289.1 \mathrm{eV}, 286.4 \mathrm{eV}$ is observed and attributed to $\mathrm{C}-\mathrm{O}, \mathrm{COOH}$, $\mathrm{C}-\mathrm{N}$ groups, respectively (Fig. $2 \mathrm{~h}^{\prime}-\mathrm{j}^{\prime}$ ); the peak at $\sim 284.8 \mathrm{eV}$ is assigned to $\mathrm{C}-\mathrm{C}$ group in all the cases. For an aromatic SAM (Fig. 2l'), a broad peak at $291.5 \mathrm{eV}$ is observed, locating at $c a$. $7 \mathrm{eV}$ higher than the $\mathrm{C}-\mathrm{C}$ peak at $\sim 284.8 \mathrm{eV}$, which can be assigned to shakeup satellite. Similar satellite peaks in C 1s XPS were observed in aromatic monolayers on metallic substrates previously. ${ }^{50,51}$ Fig. $2 \mathrm{~m}^{\prime}$ shows C $1 \mathrm{~s}$ XPS spectra, mainly composed of $-\mathrm{CH}_{2}-$ binding of SAM- $-\mathrm{CH}_{3}$. Among the $\mathrm{C} 1 \mathrm{~s}$ peaks for the $\mathrm{SAM}-\mathrm{CF}_{3}$, the peaks at around $290.9 \mathrm{eV}$ and $293.3 \mathrm{eV}$ are observed as shown in Fig. $2 n^{\prime}$, which are assigned to $-\mathrm{CF}_{2}-$ and $-\mathrm{CF}_{3}$ peaks, respectively. These results demonstrate the successful film growth of SAM-OH, SAM-COOH, SAM- $\mathrm{NH}_{2}$, $\mathrm{SAM}-\mathrm{C}_{6} \mathrm{H}_{5}, \mathrm{SAM}-\mathrm{CH}_{3}$, and $\mathrm{SAM}-\mathrm{CF}_{3}$ from gold substrates.

From AFM topographies (Fig. 20-u), almost all these surfaces exhibit a flat and homogenous conformation with a small RMS value of $\sim 1 \mathrm{~nm}$. The monolayer thickness of each surface was in the range of 1.0-1.3 nm using ellipsometry (Table S3 $\uparrow$ ). And the ratio of peak intensities of $S 2 p_{3 / 2}$ and $A u$ on each surface is in the range of 0.048-0.055 (Table S3†), indicating that the average density of the functional groups modified on each surface is almost the same. ${ }^{52}$ From all the parameters above, uniform and smooth monomolecular layers are obtained, in which surface roughness and defects probably have little effect on the Dopa binding strength.

\section{Interpretation of direct force measurements}

Fig. 3 shows typical force-distance curves for approach and retraction of a DOPA-modified cantilever from different surfaces, where positive force denotes repulsive interaction and negative force represents attractive interaction, respectively. In the case of hydrophilic surfaces (Fig. 3a), a weak attraction of DOPA-surface occurs at short range; at hydrophobic surfaces (Fig. $3 \mathrm{~b}$ ), the force becomes stronger, especially in the presence of $\mathrm{C}_{6} \mathrm{H}_{5}$-terminated surface occurring at long range. Fig. $3 \mathrm{c}$ shows the tip retracting from hydrophilic surfaces, exhibiting an increase of adhesive force with the enhancement of surface hydrophobicity from SAM-OH to bare gold. When the hydrophobicity continues to increase from $\mathrm{SAM}-\mathrm{C}_{6} \mathrm{H}_{5}$ to $\mathrm{SAM}-\mathrm{CF}_{3}$, the adhesive force decreases from a maximum attractive force at $\mathrm{C}_{6} \mathrm{H}_{5}$-terminated surface (Fig. 3d).

Further investigation on the surface forces are shown as histograms of adhesive forces in the presence of different surfaces (Fig. 4). When the DOPA-modified AFM tip separates from $\mathrm{OH}_{-}, \mathrm{COOH}-$, and $\mathrm{NH}_{2}$-terminated surfaces, the distribution of adhesive force is narrow and the peak value is around 1 $\mathrm{nN}$. With the increase of surface hydrophobicity, adhesive force reaches the maximum numerical range of $2.2-6.2 \mathrm{nN}$ in the presence of $\mathrm{C}_{6} \mathrm{H}_{5}$-terminated surface. The adhesive force of DOPA-surface becomes weaker when the hydrophobicity continues to increase, such as $\mathrm{CH}_{3^{-}}$and $\mathrm{CF}_{3}$-terminated surfaces. Previous studies showed that hydrophobic forces dominate the adhesion between hydrophobic phenylalanine residue and inorganic substrate,$^{53}$ while herein we found that the interaction strength of DOPA does not always increase with the increase of contact angle (Fig. $4 \mathrm{~h}$ ). It indicates that the hydrophobic interaction does not play a decisive role in the DOPA adhering to different wet surfaces, instead, other forms of binding are proposed to do.

\section{DLVO and EDLVO theories in modeling the approach force profiles}

In order to illustrate the contribution of DLVO interactions, the resultant forces (including van der Waals and electrostatic forces) are calculated through eqn (2)-(6) (see green lines in Fig. 5). The calculated profiles are not fitted with the experimental data, primarily attributed to the fact that the continuum theory of attractive van der Waals force and repulsive doublelayer force fails to describe the interactions between DOPAmodified AFM tips and functional surfaces as a function of separation from each other. So, an additional force, denoted as structural force, should be taken into account, which plays an important role in such cases. Via the EDLVO model (eqn (7) and (8)), the peak size of the approach curve agrees with the structural force, indicating that other kinds of interactions come into play. On the $\mathrm{OH}-, \mathrm{COOH}-$, and $\mathrm{NH}_{2}$-terminated surfaces (Fig. 5a-c), in addition to DLVO forces, a weak attractive force 
<smiles>C1CCC(CC2CC2)CC1</smiles>

(a)

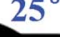

$\left.\int_{(s)}^{\text {coon coon coon }}\right|_{(\mathrm{s})} ^{\text {con }}$

(b)

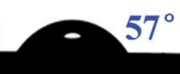

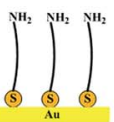

(c)
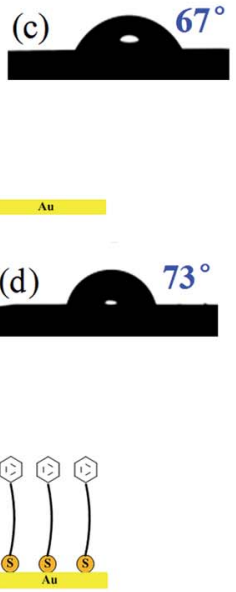

(e) $82^{\circ}$<smiles>OCC1CCCCC1</smiles>

(f) $100^{\circ}$<smiles>OCC1CCCCC1</smiles>

(g)

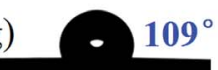

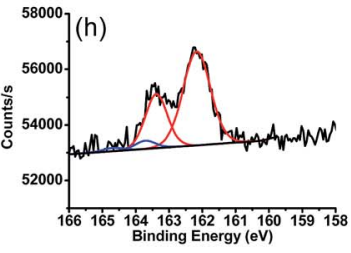
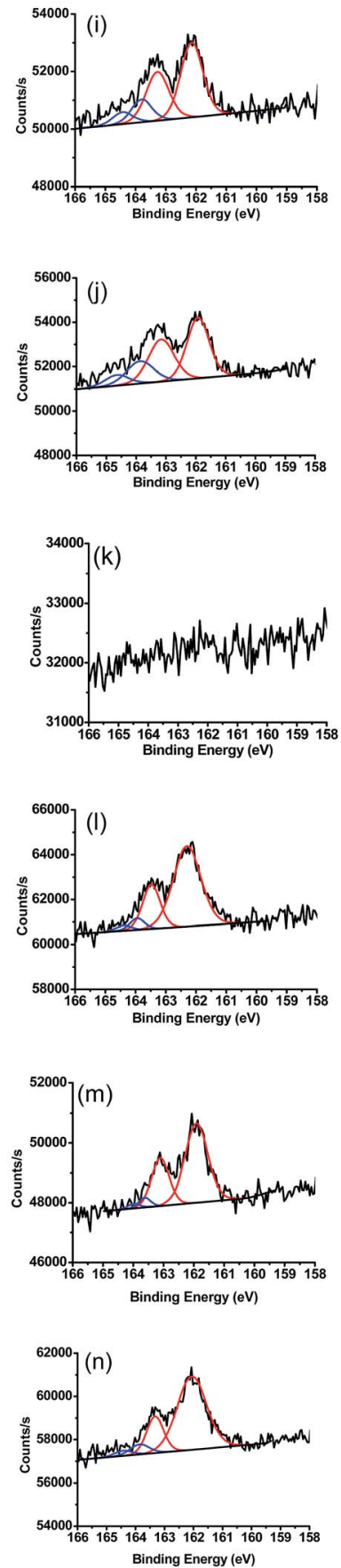
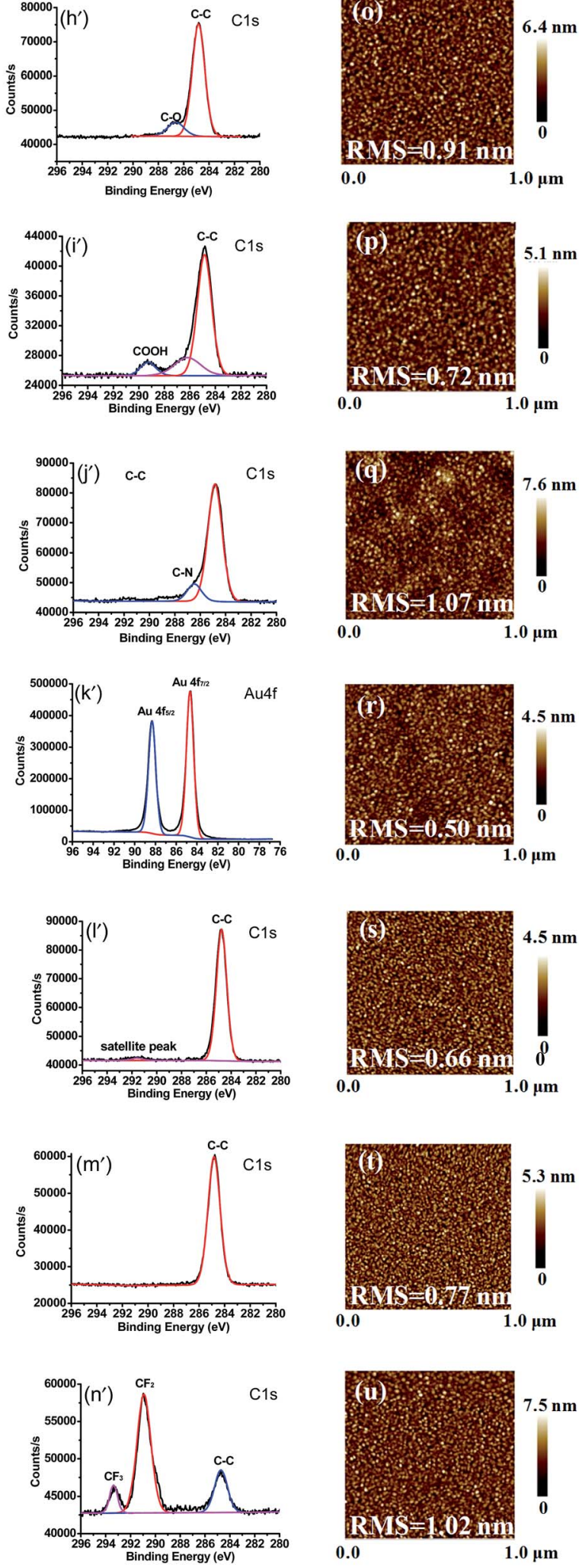

Fig. 2 CA $(a-g)$, XPS spectra $\left(h-n\right.$ and $\left.h^{\prime}-n^{\prime}\right)$, and AFM topography $(0-u)$ of bare gold surface and different substrates modified by SAM-OH, $\mathrm{SAM}-\mathrm{COOH}, \mathrm{SAM}-\mathrm{NH}_{2}, \mathrm{SAM}-\mathrm{C}_{6} \mathrm{H}_{5}, \mathrm{SAM}-\mathrm{CF}_{3}$, and $\mathrm{SAM}-\mathrm{CH}_{3}$, respectively.

appears at short-range, attributed to hydrogen bond interaction between hydroxyl groups of DOPA (hydrogen donors) and oxygen or nitrogen atoms (hydrogen acceptors) of the surface
(Fig. 6a). The electronegativity of nitrogen is weaker than oxygen, so it is considered that the binding strength of $\mathrm{N} \cdots \mathrm{H}-\mathrm{O}$ hydrogen bonds (in the case of $\mathrm{NH}_{2}$-terminated surface) is also 

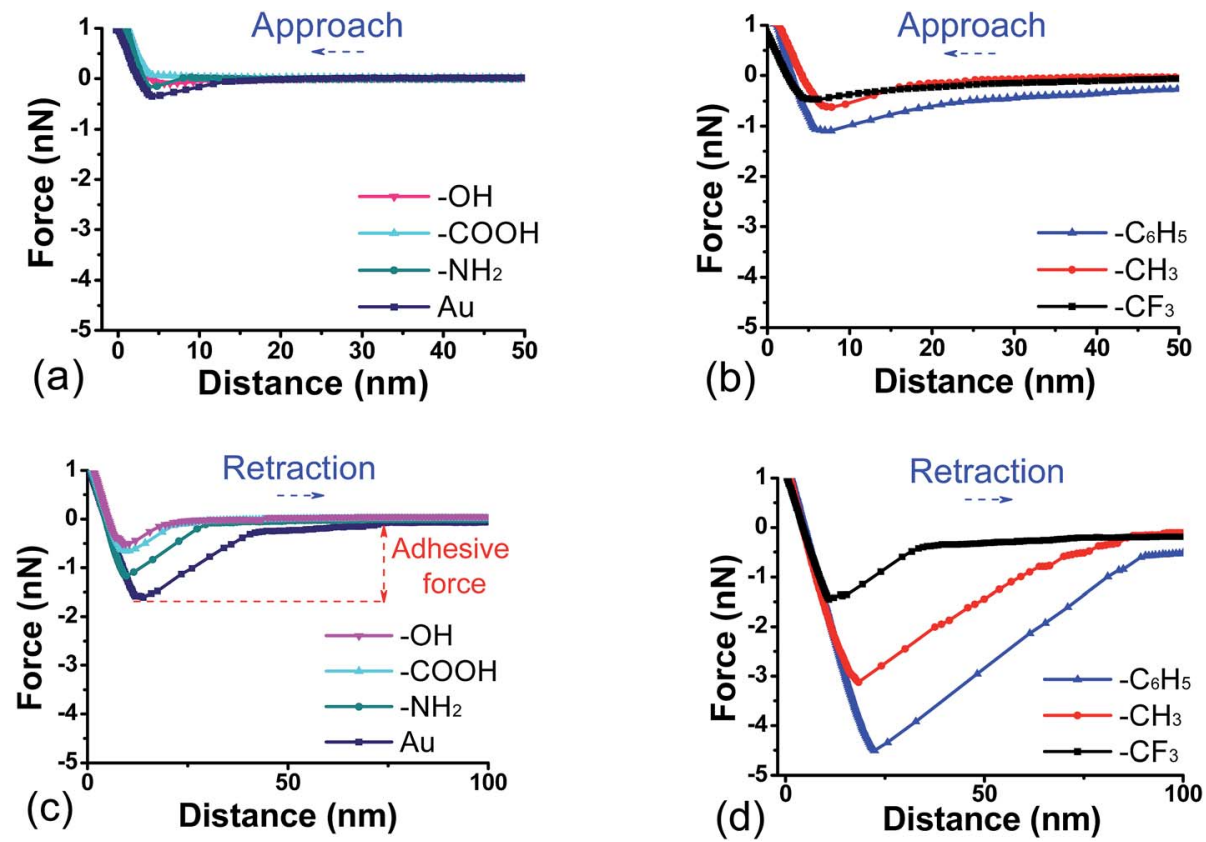

Fig. 3 Approach ( $a$ and $b$ ) and retraction ( $c$ and $d$ ) force curves as a function of separation between DOPA-modified AFM tip and functional surfaces in buffer solution.
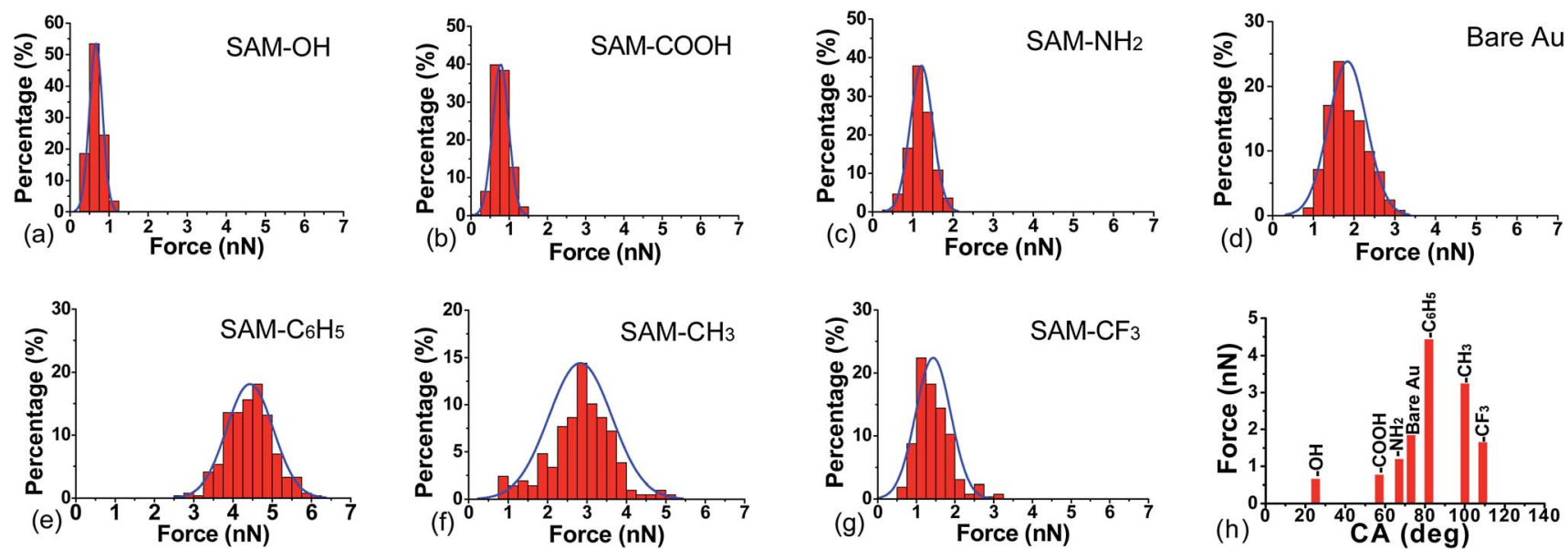

Fig. 4 Histograms of adhesion forces in the presence of (a) $\mathrm{SAM}-\mathrm{OH}$, (b) $\mathrm{SAM}-\mathrm{COOH}$, (c) $\mathrm{SAM}-\mathrm{NH}_{2}$, (d) bare gold, (e) $\mathrm{SAM}-\mathrm{C}_{6} \mathrm{H}_{5}$, (f) $\mathrm{SAM}-\mathrm{CF}_{3}$, (g) SAM- $\mathrm{CH}_{3}$ surfaces, and (h) comparison of all the average adhesive forces. Blue lines correspond to a Gaussian fit.

weaker than that of $\mathrm{O} \cdots \mathrm{H}-\mathrm{O}$ hydrogen bonds (in the case of $\mathrm{OH}-$ and $\mathrm{COOH}$-terminated surfaces). But, it is noticed that the attractive force reaches the strongest one in the presence of $\mathrm{NH}_{2}$-terminated surface among the hydrophilic surfaces, because cation $-\pi$ interaction may also be present between the charged amine groups of surface and the catechol groups of DOPA in addition to hydrogen bonds, which is considered to make a strong contribution to the attractive force. The aromatic ring and $o$-hydroxy groups in the residues of DOPA can form hydrophobic and coordination interaction with the gold surface (Fig. 5d), respectively. Compared with hydrophobic interaction, coordination plays a decisive role in the adhesion of DOPA to gold surface. ${ }^{54}$
When the surface wettability tends to be more hydrophobic, such as $\mathrm{C}_{6} \mathrm{H}_{5}$-terminated surface, the attractive force reaches a maximum value, probably due to quadrupole-quadrupole interaction between aromatic groups of DOPA and the functional surface, ${ }^{55-57}$ or both quadrupole-quadrupole and hydrophobic interactions (Fig. 6b). When the surface contact angle continues to increase, the attractive force in the case of $\mathrm{CH}_{3}$ terminated surface is dominated by the hydrophobic interaction between the aromatic ring of DOPA and the alkyl surface. By contrast, the attractive force continues to decrease on the $\mathrm{CF}_{3}$-terminated surface, because part of the DOPA molecules of the probe participate into hydrophobic interactions and part of them participate into hydrogen bonds, ${ }^{58}$ in which case 

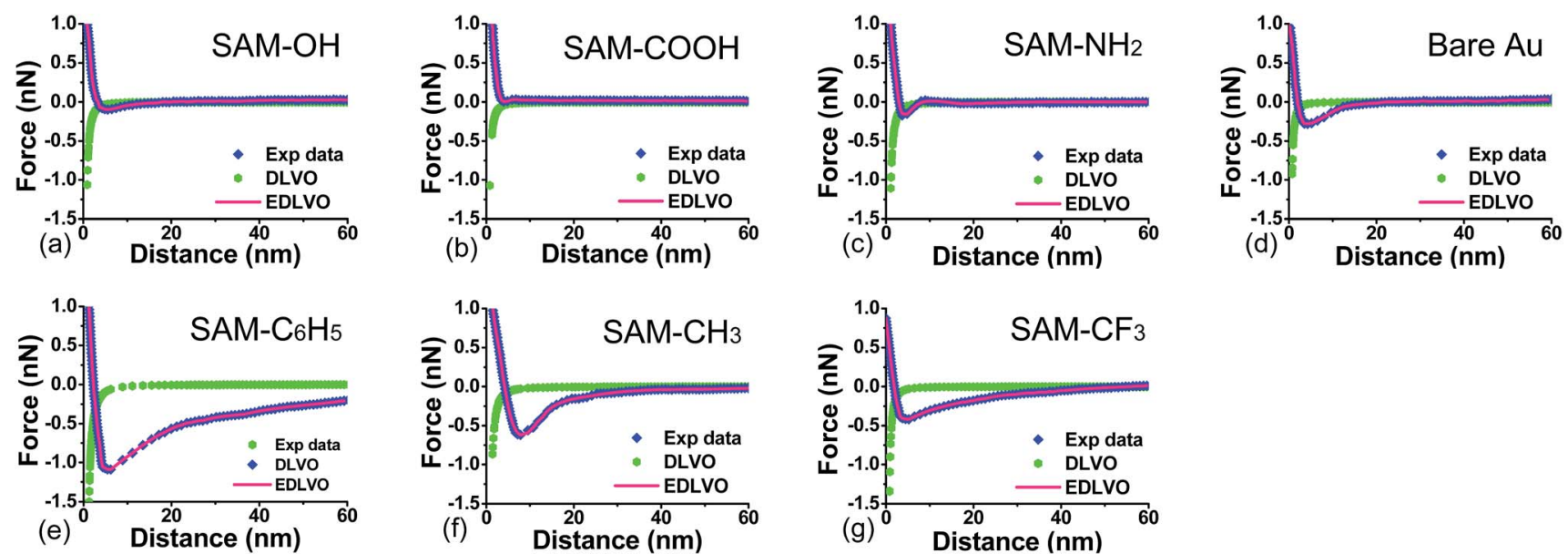

Fig. 5 Force profiles and their predictions through DLVO and EDLVO models between DOPA-modified AFM tips and functional surfaces in buffer solution.
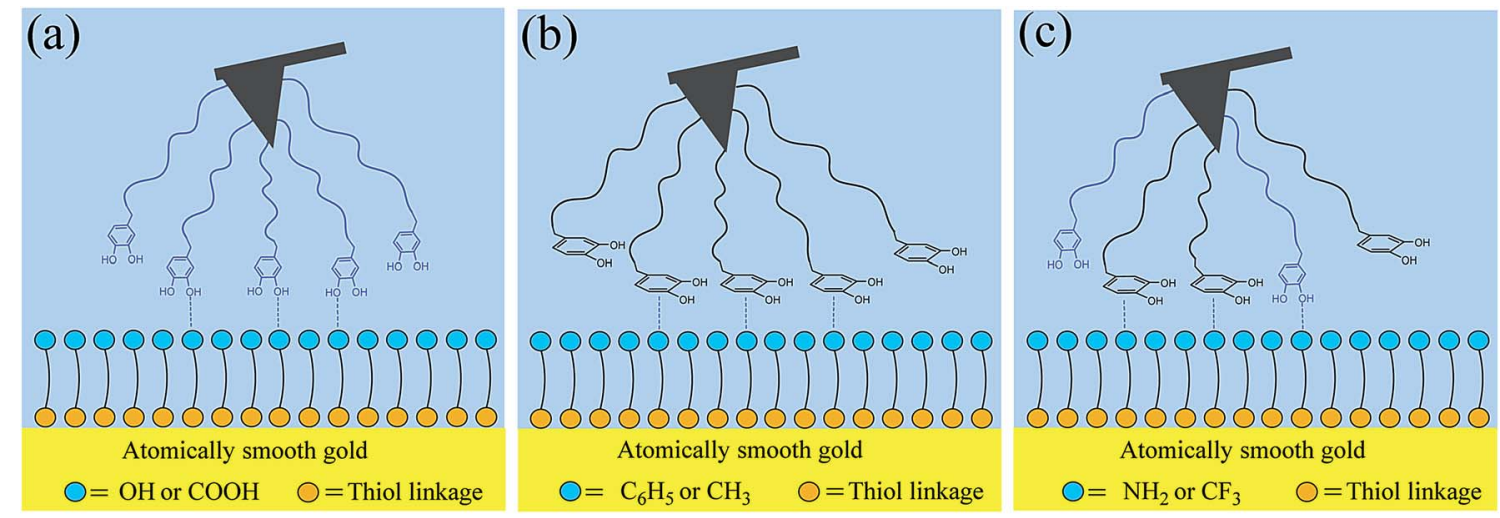

Fig. 6 Schematic representation of interactions of DOPA-surface.

hydrogen bond interaction is considered to make a weak contribution to the attractive force.

Via the extended DLVO (EDLVO) model, the fitting profiles are obtained (see pink lines in Fig. 5), with all the fitting parameters listed in the above Table S2. $\dagger$ It can be concluded that the new model successfully predicts the relationship between force and distance observed in the experimental profiles, indicating that other kinds of interactions (such as hydrophobic interaction et al.) come into play. On the $\mathrm{OH}$-, $\mathrm{COOH}$-, and $\mathrm{NH}_{2}$-terminated surface (Fig. $5 \mathrm{a}-\mathrm{c}$ ), in addition to electrostatic and van der Waals forces, a weak attractive force appears at short-range, attributed to H-bond interaction between hydroxyl groups of DOPA (hydrogen donors) and oxygen or nitrogen atoms (hydrogen acceptors) on the surface. For gold surface, the attraction is stronger, because of the hydrophobic interaction of DOPA-gold, which is similar to DOPA-hydrophobic surface interaction. When the surface wettability tends to be more hydrophobic, such as $\mathrm{C}_{6} \mathrm{H}_{5}$-terminated surface, the attractive force reaches a maximum value, due to quadrupole-quadrupole interaction between aromatic groups of DOPA and the functional surface, as well as hydrophobic interaction between aromatic group of DOPA and hydrophobic chain of the surface. It indicates that two surfaces of DOPA-modified AFM tip and functional surface contact with each other closely, which is the main reason why they tend to adhere each other during separation. When the surface contact angle continues to increase, a likely reason for the decrease of attractive force in the case of $\mathrm{CH}_{3}$-terminated surface is the single hydrophobic interaction between the aromatic ring of DOPA and the alkyl surface. By contrast, the attractive force reduces further as shown in Fig. $5 \mathrm{~g}$, because of both contributions of $\mathrm{H}$-bond and hydrophobic interactions, in which $\mathrm{H}-$ bond interaction originates from hydroxyl groups of DOPA bound to fluorine (highly electronegative atoms) of perfluorinated surface.

Herein, we prepared a series of SAM-modified gold surfaces with a variation in water wettability and surface chemistry, and investigated the interactions of DOPA with model surfaces through chemical force microscopy. It was found that surface wettability is not the single key parameter to prevent or enhance DOPA-surface adhesion, but the surface chemistry plays an important role in it. Moreover, it was found out DOPA has 
a strong ability to adhere to various surfaces, because DOPA contains catechol groups owning both of aromatic ring and $o$ hydroxy structure which can determine the adhesion behavior between DOPA and surface. For $o$-hydroxy controlled adhesion surfaces, such as $\mathrm{OH}^{-}$and $\mathrm{COOH}$-terminated surfaces, the aromatic ring of DOPA is proposed to have a perpendicular orientation with the surface as shown in Fig. 6a; for aromatic ring controlled adhesion surfaces, such as $\mathrm{CH}_{3}{ }^{-}$and $\mathrm{C}_{6} \mathrm{H}_{5^{-}}$ terminated surfaces, the aromatic ring is suggested to have a parallel orientation with the surface as shown in Fig. 6b. For the SAM- $\mathrm{C}_{6} \mathrm{H}_{5}$ surface, it does not exhibit the strongest hydrophobic property while exhibits the strongest adhesion of DOPAsurface among all the modified surfaces, because there may be a superposition resulting from two kinds of binding forces of quadrupole-quadrupole and hydrophobic interactions. While in the case of SAM-NH be a mixed superposition configuration, because DOPA residues can interact with surface in perpendicular or parallel, respectively, as shown in Fig. 6c. Among the hydrophilic surfaces, the attractive force reaches the strongest one in the case of $\mathrm{NH}_{2}-$ terminated surface, probably because catechol groups can form hydrogen bonding or cation- $\pi$ interaction with the surface. Among the hydrophopbic surfaces, the attractive force reaches the weakest one in the case of $\mathrm{CF}_{3}$-terminated surface, because catechol groups can form hydrophobic interaction or hydrogen bonding with the surface, and each of the catechol groups cannot take part in the two interactions at the same time.

\section{Conclusions}

We developed a novel approach to investigate the interactions between DOPA and specific functional group surfaces with variable wettability. When the DOPA-terminated tip retracted from each surface, the adhesion increases with the enhancement of surface hydrophobicity from SAM-OH to SAM- $\mathrm{C}_{6} \mathrm{H}_{5}$; when the surface hydrophobicity continues to increase, the adhesion gradually decreases, indicating that the hydrophobic interaction does not play a decisive role, instead, other forms of binding play a key role. We found that DOPA can adhere to different functional surfaces, because aromatic ring or $o$ hydroxy structure of catechol group can separately dominate the adhesion of DOPA-surface. It was proposed two kinds of configurations of the DOPA residue on the surface: one is the plane of the aromatic ring being perpendicular on the surface and binding to it via o-hydroxy of catechol, such as $\mathrm{OH}^{-}$and $\mathrm{COOH}$-terminated surfaces, and the other is the plane of the aromatic ring being parallel to the surface and binding to it via aromatic ring of catechol, such as $\mathrm{C}_{6} \mathrm{H}_{5^{-}}$and $\mathrm{CH}_{3}$-terminated surfaces. Furthermore, there may be two forms of binding in a single configuration, such as $\mathrm{SAM}-\mathrm{C}_{6} \mathrm{H}_{5}$ surface, due to a superposition of two kinds of binding forces (quadrupolequadrupole and hydrophobic interactions). For $\mathrm{NH}_{2}-$ and $\mathrm{CF}_{3}-$ terminated surfaces, two kinds of configurations of the DOPA residue were proposed on the surface, separately. DOPA exhibits a stronger adhesion to the $\mathrm{NH}_{2}$-terminated surface than that to other hydrophilic surfaces, due to cation- $\pi$ interaction which is considered to make a strong contribution to the attractive force.
While, DOPA exhibits a weakest adhesion to the $\mathrm{CF}_{3}$-terminated surface than that to other hydrophobic surfaces, due to hydrogen bond interaction which is considered to make a weak contribution to the attractive force. Compared with surface wettability, surface chemical composition determines different types of DOPA-surface interactions, being a key factor in DOPA adhesion process. This research can be applied to the design and fabrication of adhesion or anti-adhesion materials.

\section{Conflict of interest}

The authors declare no competing financial interest.

\section{Acknowledgements}

This work was funded by the projects of PetroChina Exploration and Development Research Institute (2015-40222-000006 and RIPED-2017-JS-88), the Important National Science and Technology Specific Project of China (2017ZX05013003004 and 2016ZX05025003009), and the National Natural Science Foundation of China (21603240).

\section{References}

$1 \mathrm{H}$. G. Silverman and F. F. Roberto, Understanding marine mussel adhesion, Mar. Biotechnol., 2007, 9(6), 661-681.

2 H. J. Cha, D. S. Hwang and S. Lim, Development of bioadhesives from marine mussels, Biotechnol. J., 2008, 3(5), 631-638.

3 D. J. Crisp, G. Walker, G. A. Young and A. B. Yule, Adhesion and substrate choice in mussels and barnacles, J. Colloid Interface Sci., 1985, 104(104), 40-50.

4 J. H. Waite, Adhesion a la moule, Integr. Comp. Biol., 2002, 42(6), 1172-1180.

5 M. A. Even, J. Wang and Z. Chen, Structural information of mussel adhesive protein mefp-3 acquired at various polymer/mefp-3 solution interfaces, Langmuir, 2008, 24(11), 5795-5801.

$6 \mathrm{~J}$. H. Waite and M. L. Tanzer, Polyphenolic substance of mytilus edulis: novel adhesive containing L-Dopa and hydroxyproline, Science, 1981, 212(4498), 1038-1040.

7 J. H. Waite, N. H. Andersen, S. Jewhurst and C. J. Sun, Mussel adhesion: finding the tricks worth mimicking, J. Adhes., 2005, 81(3-4), 297-317.

8 K. G. Neoh and E. T. Kang, Combating bacterial colonization on metals via polymer coatings: relevance to marine and medical applications, ACS Appl. Mater. Interfaces, 2011, 3(8), 2808-2819.

9 J. Ren, P. P. Han, H. L. Wei and L. Y. Jia, Fouling-resistant behavior of silver nanoparticle-modified surfaces against the bioadhesion of microalgae, ACS Appl. Mater. Interfaces, 2014, 6(6), 3829-3838.

10 M. Donkerwolcke, F. Burny and D. Muster, Tissues and bone adhesives-historical aspects, Biomaterials, 1998, 19(16), 1461-1466.

11 B. J. Kim, D. X. Oh, S. Kim, J. H. Seo, D. S. Hwang, A. Masic, D. K. Han and H. J. Cha, Mussel-mimetic protein-based 
adhesive hydrogel, Biomacromolecules, 2014, 15(5), 15791585.

12 R. Y. Floriolli, L. J. von and J. H. Waite, Marine surfaces and the expression of specific byssal adhesive protein variants in mytilus, Mar. Biotechnol., 2000, 2(4), 352-363.

$13 \mathrm{~J}$. H. Waite and X. X. Qin, Polyphenolic polyphosphoprotein from the adhesive pads of mytilus edulis, Biochemistry, 2001, 40(9), 2887-2893.

14 H. Lee, N. F. Scherer and P. B. Messersmith, Single-molecule mechanics of mussel adhesion, Proc. Natl. Acad. Sci. U. S. A., 2006, 103(35), 12999-13003.

15 V. V. Papov, T. V. Diamond, K. Biemann and J. H. Waite, Hydroxyarginine-containing polyphenolic proteins in the adhesive plaques of the marine mussel mytilus edulis, $J$. Biol. Chem., 1995, 270(34), 20183-20192.

16 N. R. Martinez Rodriguez, S. Das, Y. Kaufman, J. N. Israelachvili and J. H. Waite, Interfacial $\mathrm{pH}$ during mussel adhesive plaque formation, Biofouling, 2014, 31(2), 221-227.

17 M. Yu and T. J. Deming, Synthetic polypeptide mimics of marine adhesives, Macromolecules, 1998, 31(15), 4739-4745.

$18 \mathrm{M}$. Yu, J. H. Wang and T. J. Deming, Role of L-3,4dihydroxyphenylalanine in mussel adhesive proteins, J. Am. Chem. Soc., 1999, 121(24), 5825-5826.

19 Q. Lin, D. Gourdon, C. Sun, N. Holten-Andersen, T. H. Andersen, J. H. Waite and J. N. Israelachvili, Adhesion mechanisms of the mussel foot proteins mfp-1 and mfp-3, Proc. Natl. Acad. Sci. U. S. A., 2007, 104(10), 3782-3786.

20 J. J. Wang, M. N. Tahir, M. Kappl, W. Tremel, N. Metz, M. Barz, P. Theato and H. J. Butt, Influence of binding-site density in wet bioadhesion, Adv. Mater., 2008, 20(20), 3872-3875.

21 Z. A. Levine, M. V. Rapp, W. Wei, R. G. Mullen, C. Wu, G. H. Zerze, J. Mittal, J. H. Waite, J. N. Israelachvili and J. E. Shea, Surface force measurements and simulations of mussel-derived peptide adhesives on wet organic surfaces, Proc. Natl. Acad. Sci. U. S. A., 2016, 113(16), 4332-4337.

22 Y. R. Li, M. Qin, Y. Li, Y. Cao and W. Wang, Single molecule evidence for the adaptive binding of DOPA to different wet surfaces, Langmuir, 2014, 30(15), 4358-4366.

23 D. S. Hwang, M. J. Harrington, Q. Lu, A. Masic, H. Zeng and J. H. Waite, Mussel Foot Protein-1 (Mcfp-1) Interaction with Titania Surfaces, J. Mater. Chem., 2012, 22(31), 15530-15533.

24 J. Yu, W. Wei, E. Danner, J. N. Israelachvili and J. H. Waite, Effects of Interfacial Redox in Mussel Adhesive Protein Films on Mica, Adv. Mater., 2011, 23(20), 2362.

25 J. M. Curran, R. Chen and J. A. Hunt, The Guidance of Human Mesenchymal Stem Cell Differentiation in Vitro by Controlled Modifications to the Cell Substrate, Biomaterials, 2006, 27(27), 4783-4793.

26 J. Yu, Y. Kan, M. Rapp, E. Danner, W. Wei, S. Das, D. R. Miller, Y. Chen, J. H. Waite and J. N. Israelachvili, Adaptive hydrophobic and hydrophilic interactions of mussel foot proteins with organic thin films, Proc. Natl. Acad. Sci. U. S. A., 2013, 110(39), 15680-15685.
27 Q. Lu, E. Danner, J. H. Waite, J. N. Israelachvili, H. Zeng and D. S. Hwang, Adhesion of mussel foot proteins to different substrate surfaces, J. R. Soc., Interface, 2013, 10(79), 20120759.

28 E. W. Danner, Y. J. Kan, M. U. Hammer, J. N. Israelachvili and J. H. Waite, Adhesion of mussel foot protein mefp-5 to mica: an underwater superglue, Biochemistry, 2012, 51(33), 6511-6518.

29 D. S. Hwang, M. J. Harrington, Q. Y. Lu, A. Masic, H. B. Zeng and J. H. Waite, Mussel foot protein-1 (mcfp-1) interaction with titania surfaces, J. Mater. Chem., 2012, 22(31), 1553015533.

30 J. Yu, W. Wei, M. S. Menyo, A. Masic, J. H. Waite and J. N. Israelachvili, Adhesion of mussel foot protein-3 to $\mathrm{TiO}_{2}$ surfaces: the effect of pH, Biomacromolecules, 2013, 14(4), 1072-1077.

31 H. B. Zeng, D. S. Hwang, J. N. Israelachvili and J. H. Waite, Strong reversible $\mathrm{Fe}^{3+}$-mediated bridging between DOPAcontaining protein films in water, Proc. Natl. Acad. Sci. U. S. A., 2010, 107(29), 12850-12853.

32 W. Wei, J. Yu, C. Broomell, J. N. Israelachvili and J. H. Waite, Hydrophobic enhancement of DOPA-mediated adhesion in a mussel foot protein, J. Am. Chem. Soc., 2013, 135(1), 377383.

33 B. Sivaraman and R. A. Latour, The relationship between platelet adhesion on surfaces and the structure versus the amount of adsorbed fibrinogen, Biomaterials, 2010, 31(31), 832-839.

34 J. M. Curran, R. Chen and J. A. Hunt, The guidance of human mesenchymal stem cell differentiation in vitro by controlled modifications to the cell substrate, Biomaterials, 2006, 27(27), 4783-4793.

35 B. Sivaraman, K. P. Fears and R. A. Latour, Investigation of the effects of surface chemistry and solution concentration on the conformation of adsorbed proteins using an improved circular dichroism method, Langmuir, 2009, 25(5), 3050-3056.

36 H. L. Cao, X. Y. Liu, F. H. Meng and P. K. Chu, Biological actions of silver nanoparticles embedded in titanium controlled by micro-galvanic effects, Biomaterials, 2011, 32(3), 693-705.

37 J. Yu, E. Danner, J. N. Israelachvili and J. H. Waite, Effects of interfacial redox in mussel adhesive protein films on mica, Adv. Mater., 2014, 23(20), 2362-2366.

38 J. Israelachvili, Interfacial forces, J. Vac. Sci. Technol., A, 1992, 10(5), 2961-2971.

39 Y. Liang, N. Hilal, P. Langston and V. Starov, Interaction forces between colloidal particles in liquid: theory and experiment, Adv. Colloid Interface Sci., 2007, 134-135(21), 151-166.

$40 \mathrm{~J}$. Gregory, Approximate expressions for retarded van der waals interaction, J. Colloid Interface Sci., 1981, 83(1), 138145.

$41 \mathrm{~J}$. N. Israelachvili, Van der waals forces between particles and surfaces, Intermolecular and surface forces, Elsevier, Amsterdam, 3rd edn, 2011, ch. 13, pp. 253-289. 
42 J. N. Israelachvili, K. Kristiansen, M. A. Gebbie, D. W. Lee, S. H. Donaldson Jr, S. Das, M. V. Rapp, X. Banquy, M. Valtiner and J. Yu, The intersection of interfacial forces and electrochemical reactions, J. Phys. Chem. B, 2013, 117(51), 16369-16387.

43 R. Hogg, T. W. Healy and D. W. Fuerstenau, Mutual coagulation of colloidal dispersions, Trans. Faraday Soc., 1966, 62, 1638-1651.

$44 \mathrm{~J}$. N. Israelachvili, Electrostatic forces between surfaces in liquids, Intermolecular and surface forces, Elsevier, Amsterdam, 3rd edn, 2011, ch. 14, pp. 291-340.

45 H. J. Butt, Measuring electrostatic, van der waals, and hydration forces in electrolyte solutions with an atomic force microscope, Biophys. J., 1991, 60(6), 1438-1444.

$46 \mathrm{H}$. Guo, A simple algorithm for fitting a Gaussian function, IEEE Signal Process. Mag., 2011, 28(5), 134-137.

47 J. Z. Wu, F. H. Liu, G. Chen, X. Wu, D. S. Ma, W. Zhang, H. Yang and J. B. Wang, Effect of ionic strength on the interfacial forces between oil/brine/rock interfaces: a chemical force microscopy study, Energy Fuels, 2016, 30(1), 273-280.

48 H. Wang, S. F. Chen, L. Y. Li and S. Y. Jiang, Improved method for the preparation of carboxylic acid and amine terminated self-assembled monolayers of alkanethiolates, Langmuir, 2005, 21(7), 2633-2636.

49 P. M. Dietrich, N. Graf, T. Gross, A. Lippitz, B. Schupbach, A. Bashir, C. Woll, A. Terfort and W. E. Unger, Selfassembled monolayers of aromatic $\omega$-aminothiols on gold: surface chemistry and reactivity, Langmuir, 2010, 26(6), 3949-3954.

50 D. Nilsson, S. Watcharinyanon, M. Eng, L. Li, E. Moons, L. S. O. Johansson, M. Zharnikov, A. Shaporenko, B. Albinsson and J. Martensson, Characterization of self- assembled monolayers of oligo(phenyleneethynylene) derivatives of varying shapes on gold: effect of laterally extended pi-systems, Langmuir, 2007, 23(11), 6170-6181.

51 Y. Harada, T. Koitaya, K. Mukai, S. Yoshimoto and J. Yoshinobu, Spectroscopic characterization and transport properties of aromatic monolayers covalently attached to $\mathrm{Si}$ (111) surfaces, J. Phys. Chem. C, 2013, 117(15), 7497-7505.

52 D. Y. Petrovykh, H. Kimurasuda, M. J. Tarlov and L. J. Whitman, Quantitative characterization of DNA films by X-ray photoelectron spectroscopy, Langmuir, 2004, 20(2), 429-440.

53 Y. Razvag, V. Gutkin and M. Reches, Probing the interaction of individual amino acids with inorganic surfaces using atomic force spectroscopy, Langmuir, 2013, 29(32), 1010210109.

54 A. Ooka and R. L. Garrell, Surface-enhanced Raman spectroscopy of DOPA-containing peptides related to adhesive protein of marine mussel, mytilus edulis, Biopolymers, 2000, 57(2), 92-102.

55 S. K. Burley and G. A. Petsko, Weakly polar interactions in proteins, Adv. Protein Chem., 1988, 39(2), 125-189.

56 P. D. M. Hatfield, Y. N. Palermo, J. Csontos, F. R. Murphy and S. Lovas, Quantum Chemical quantification of weakly polar interaction energies in the TC5b miniprotein, J. Phys. Chem. $B, 2008,112(11), 3503-3508$.

57 M. J. Plevin, D. L. Bryce and J. Boisbouvier, Direct detection of $\mathrm{CH} /$ pi interactions in proteins, Nat. Chem., 2010, 2(6), 466-471.

58 S. R. Chaudhari, S. Mogurampelly and N. Suryaprakash, Engagement of $\mathrm{CF}_{3}$ group in $\mathrm{N}-\mathrm{H} \cdots \mathrm{F}-\mathrm{C}$ hydrogen bond in the solution state: NMR spectroscopy and MD simulation studies, J. Phys. Chem. B, 2013, 117(4), 1123-1129. 